\title{
Assessment of spontaneous baroreflex sensitivity in neonates
}

Emmanuel Drouin, Véronique Gournay, Jean Calamel, Alain Mouzard, Jean-Christophe Rozé

\begin{abstract}
Aims-To determine whether it is possible to assess baroreflex sensitivity in neonates by studying only spontaneous variations in systolic blood pressure and heart rate. Methods-ECG and non-invasive blood pressure signals were continuously studied in 14 preterm neonates (term 29-32 weeks) and five term neonates (term 40-41 weeks). Non-invasive blood pressure measures were obtained using a Finapres placed around the child's wrist. Both signals (ECG and blood pressure), sampled at $400 \mathrm{~Hz}$, were digitised by an A/D converter and stored in a binary mode on magnetic disk. An inhouse software QRS detection algorithm was used to define $R$ peaks of the QRS complexes with an accuracy greater than 2 ms. Four 4 minute periods were recorded in each infant. The slope of the linear regression of RR intervals versus systolic blood pressure was calculated in each period and the mean value of the four slopes was then considered as the index of baroreflex sensitivity (in $\mathrm{ms} / \mathrm{mm} \mathrm{Hg}$ ) in each neonate.

Results-Spontaneous baroreflex sensitivity was lower in preterm neonates than in term neonates (mean(SD): 4.07 (2.19) $\mathrm{ms} / \mathrm{mm} \mathrm{Hg}$ vs 10.23 (2.92) $\mathrm{ms} / \mathrm{mm} \mathrm{Hg}$ ). Conclusion-Baroreflex sensitivity can be assessed in term and preterm neonates by studying spontaneous variations in systolic blood pressure alone. This method could be useful for studying the ontogeny of baroreflex sensitivity and might therefore provide information about the maturation of the autonomic nervous system. (Arch Dis Child 1997;76:F108-F112)
\end{abstract}

Keywords: blood pressure; heart rate; baroreflex sensitivity; autonomic nervous system.

Arterial baroreceptors have an important role in beat-to-beat modulation of efferent cardiovascular autonomic activity, acting on the vasculature and the heart. The baroreflex acts as a negative feedback control loop of arterial blood pressure and exerts a buffering influence on its spontaneous fluctuations.

Baroreflex sensitivity is defined as the slope of linear regression of $\mathrm{RR}$ intervals on the electrocardiogram vs systolic blood pressure. The steeper the slope, the higher the baroreflex sensitivity. A steep slope of regression line is interpreted as indicating a strong vagal reflex; a flat slope indicates a weak vagal reflex and high reflex sympathetic activity.

In adults the usual methods of measurement of baroreflex sensitivity require pharmacologically or mechanically induced changes in systolic blood pressure. These methods are limited by their perturbational character and by possible modifications of barosensitive areas induced by the drugs. ${ }^{23}$ These limitations prompted the development of another method that evaluates baroreflex sensitivity from spontaneous changes in systolic blood pressure (Sp-SBP). The spontaneous baroreflex sensitivity (Sp-BRS) method provides a reliable, non-invasive assessment of vagal cardiac baroreflex sensitivity in animals and adults within its physiological operating range. ${ }^{4}$

In human neonates, little or no information is known about baroreflex sensitivity for several reasons. First, it would be dangerous and unethical to alter pharmacologically baroreceptor activity. Second, until recently, continuous non-invasive recording of systolic blood pressure was not feasible in neonates. Automated devices using the principle of oscillometry (Dinamap; Critikon) measure arterial blood pressure non-invasively, but it is not possible to observe the instantaneous variations because the delay of the response is too long. However, we demonstrated recently that Finapres (Ohmeda), a non-invasive method displaying continuous arterial waveform of the blood pressure, displayed arterial blood pressure values reasonably close to those obtained invasively in neonates. ${ }^{6}$ Indeed, in eight neonates we compared the ability of Finapres to reproduce the beat-to-beat signal of arterial blood pressure with that of an umbilical intraarterial catheter. The agreement between the two methods was acceptable, as shown by reasonably small differences (1.81 (3.3) $\mathrm{mm} \mathrm{Hg}$ for systolic blood pressure and $0.11(1.9) \mathrm{mm}$ $\mathrm{Hg}$ for disatolic blood pressure).

The purpose of this study was, therefore, to determine whether baroreflex sensitivity can be assessed non-invasively in human neonates by studying spontaneous variations in systolic blood pressure using Finapres.

\section{Methods}

The study protocol was approved by the university hospital ethics commmittee. Eighteen preterm neonates and seven term neonates, admitted to intensive care, were studied. All infants were in a stable condition while supine. A reliable baroreflex sensitivity value could be obtained in only 19 of these 25 
Table 1 Characteristics of 19 neonates studied, values are mean (SD) and range

\begin{tabular}{lllc}
\hline & Birth weight $(g)$ & Gestational age (weeks) & Postnatal age (days) \\
\hline Preterm $(\mathrm{n}=14)$ & $1330(148)(790-2140)$ & $31.3(3.8)(26-39)$ & $17.8(21.2)(1-70)$ \\
Term $(\mathrm{n}=5)$ & $3612(415.2)(3270-4260)$ & $40.6(0.6)(40-41)$ & $3.8(1.1)(3-5)$ \\
\hline
\end{tabular}

neonates (14 preterm and five term neonates) (table 1). Assessments were performed between 0800 and 1400 hours.

ASSESSMENT OF BAROREFLEX SENSITIVITY

We recorded continuously the systolic blood pressure and the ECG signal during four 4 minute periods in each neonate. About 10 minutes was allowed to elapse between each period. In each 4 minute period, we selected a shorter period (duration of a few seconds), where systolic blood pressure varied enough to induce a heart rate response consistent with baroreflex activity-that is, bradycardia in response to an increase in systolic blood pressure $(+R R /+\Delta S B P)$ or tachycardia in response to a decrease $(-\mathrm{RR} /-\Delta \mathrm{SBP})$. This period was composed of at least 10 points (10 cardiac cycles). Baroreflex sensitivity was measured from the data recorded during this short period. We therefore obtained four measurements of baroreflex sensitivity in each infant. We considered the data suitable for baroreflex sensitivity assessment only if at least three measurements out of four produced similar results and if the regression line comprised a minimum of 10 points $(10$ cardiac cycles) with an $r \geq 0.80$. In each infant the mean value of the three or four accepted slopes was regarded as the index of baroreflex sensitivity.

\section{Methods of measurement}

During each manipulation, the electrocardiographic lead with the greatest QRS amplitude and the shortest QRS duration (usually DII) was continuously monitored using an ECG monitor (Mingograf 34; Siemens). A continuous non-invasive arterial blood pressure signal was measured using the volume clamp method and a Finapres 2300 (Ohmeda Inc, USA). The plethysmographic cuff is placed around the neonate's wrist instead of the finger, which is the usual position in adults (there were no complications or discomfort due to the use of the apparatus), and the cuff pressure was modulated to maintain transmural pressure at effectively zero. To do so, the cuff is inflated automatically to the pressure level at which the pulse volume curve is obtained with maximal amplitude (this value is usually equal to mean arterial pressure). The cuff pressure is then changed rapidly by a servocontrol system to keep the finger pulse signals constant - to keep transmural pressure across the arterial wall at zero throughout the whole cardiac cycle. Care were taken to measure blood pressure at heart level (cuff at the same hydrostatic level).

The servo-reset mode of the Finapres was turned off during the recording and was reset between recordings. The ECG and arterial blood pressure were recorded simultaneously and converted from analogue to digital (A/D) format with a temporal resolution of 200 $\mathrm{Hz} /$ channel and an amplitude resolution of 12 bits.

Sp-BRS ( $\mathrm{ms} / \mathrm{mm} \mathrm{Hg}$ ) was evaluated from Sp-SBP $( \pm \Delta$ SBP) and heart rate changes ( \pm $\triangle R R)$. Baroreflex sensitivity can be measured by computing the slope of the regression line between changes in systolic blood pressure and the following pulse interval, during either bradycardia/hypertension $(+\Delta R R /+\Delta S B P)$ or tachycardia/hypotension $(-\Delta R R /-\Delta S B P)$. Sequences of increasing or decreasing systolic pressures with directionally opposite changes in $\mathrm{RR}$ interval (+RR/- $\Delta \mathrm{SBP} ;-\mathrm{RR} /+\Delta \mathrm{SBP})$ were not analysed as they do not seem to represent physiological baroreflex responses. ${ }^{4}$ The $\mathrm{A} / \mathrm{D}$ converted signals were stored in a computer. An inhouse software QRS detection algorithm was used to define $R$ peaks of the QRS complexes with an accuracy of more than 2 ms. All data acquisitions and analyses were performed using a menu-driven software package. For calculation of Sp-BRS, a short period was selected where changes in systolic blood pressure ranged between 10 to $20 \%$ of the baseline level with concomitant changes in RR intervals. Beat-to-beat values of $R R$ intervals were plotted against systolic blood pressure values of the preceding cardiac cycle $\left(\mathrm{RR}_{(\mathrm{n}+1)} v \mathrm{~s}\right.$ $\left.\mathrm{SBP}_{n}\right)$ in a period with an increase $(+\Delta \mathrm{SBP})$ or decrease $(-\triangle S B P)$ in systolic blood pressure. A linear regression analysis between $\mathrm{RR}_{(\mathrm{n}+1)}$ and $\mathrm{SBP}_{n}$ was performed. The slope of the regression line and the corresponding correlation coefficient ( $r$ ) were calculated.

Data were expressed as mean (SD) of the mean and were compared using a paired or unpaired Student $t$ test, as applicable. Differences were considered significant at $\mathrm{P}<0.05$.

\section{Results}

The gestational ages of the preterm and term neonates were, respectively, 31.3 (3.8) and $40.60(0.550)$ weeks. Of the 18 preterm neonates studied, only 14 had enough spontaneous variations in systolic blood pressure to allow Sp-BRS to be measured. An example of Sp-BRS calculation from spontaneous beat-tobeat correlation between $\triangle \mathrm{SBP}$ and $\triangle \mathrm{RR}$ is shown in fig 1 . In these 14 preterm neonates the mean value of the $\mathrm{Sp}$-BRS was 4.07 (2.19) $\mathrm{ms} / \mathrm{mm} \mathrm{Hg}$ (table 2). Values of Sp-BRS measured during episodes of bradycardia/ hypertension $(+\Delta \mathrm{RR} /+\Delta \mathrm{SBP})$ were similar to the values measured during episodes of tachycardia/hypotension $(-\Delta \mathrm{RR} /-\Delta \mathrm{SBP})$ : respectively, $3.96(2.50)$ and $4.15(1.97) \mathrm{ms} / \mathrm{mm}$ $\mathrm{Hg}$ (table 2). This similarity is illustrated in one neonate by the similar slopes in the upper right (B) quadrant and the bottom left quadrant (A) in fig $1 \mathrm{~B}$. Out of seven term neonates, we were able to measure baroreflex sensitivity in five. The mean value of $\mathrm{Sp}$-BRS was greater than in preterm neonates (10.23 (2.92); $\mathrm{P}<0.001$ vs preterm neonates) (table 2 ). As in the preterm neonates, Sp-BRS calculated during episodes of bradycardia/hypertension $(+\Delta R R /+\Delta S B P)$ were similar to that calculated during episodes of tachycardia/hypotension $(-\Delta \mathrm{RR} /-\Delta \mathrm{SBP})$ : re- 
Table 2 Baroreflex sensitivity values in the preterm and term neonates (values are mean (SD))

\begin{tabular}{|c|c|c|c|c|c|}
\hline & No of measurements & $S p-B R S(m s / m m ~ H g)$ & $r$ & $\triangle R R$ (seconds) & $\triangle S B P(m m ~ H g)$ \\
\hline \multicolumn{6}{|l|}{ Preterm $(n=14)$ : } \\
\hline Total & 42 & $4.07(2.19)$ & $0.86(0.05)$ & & \\
\hline$+\Delta \mathrm{RR} /+\Delta \mathrm{SBP}$ & 19 & $3.96(2.50)$ & $0.83(0.03)$ & $0.04(0.02)$ & $9.16(4.20)$ \\
\hline$-\Delta \mathrm{RR} /-\Delta \mathrm{SBP}$ & 23 & $4.15(1.97)$ & $0.88(0.05)$ & $-0.04(0.02)$ & $-9.17(5.15)$ \\
\hline \multicolumn{6}{|l|}{ Term $(n=5)$ : } \\
\hline Total & 15 & $10.23(2.92)^{\star}$ & $0.86(0.06)$ & & \\
\hline$+\Delta \mathrm{RR} /+\Delta \mathrm{SBP}$ & 7 & $10.51(3.08)^{\star}$ & $0.85(0.06)$ & $0.125(0.20)$ & $5.90(6.43)$ \\
\hline$-\Delta \mathrm{RR} /-\Delta \mathrm{SBP}$ & 8 & $10.32(2.95)^{\star}$ & $0.84(0.08)$ & $-0.041(0.021)$ & $-3.75(2.30)$ \\
\hline
\end{tabular}

spectively, 10.51 (3.07) and 10.32 (2.95) $\mathrm{ms} / \mathrm{mm} \mathrm{Hg}$.

To evaluate the individual reproducibility of baroreflex sensitivity measurement, in four neonates, we measured baroreflex sensitivity at the same time of the day for four consecutive days (fig 2). Values of Sp-BRS were very similar when calculated for four consecutive days in the same four neonates at the same time in the morning. In each of the four neonates and for each point of the figure, the mean value of six accepted slopes $(r>0.8)$ was regarded as the index of Sp-BRS.

$\mathrm{Sp}$-BRS is positively correlated with the corrected age $(r=0.75, \mathrm{P}<0.001)$. Thus baroreflex sensitivity steadily increases from preterm to term neonates.

In two neonates, we measured baroreflex sensitivity before and after treatment with prantal, a parasympatholytic agent. These

A

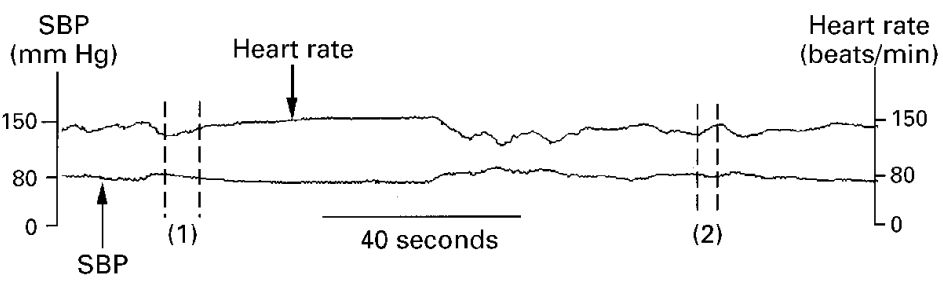

B

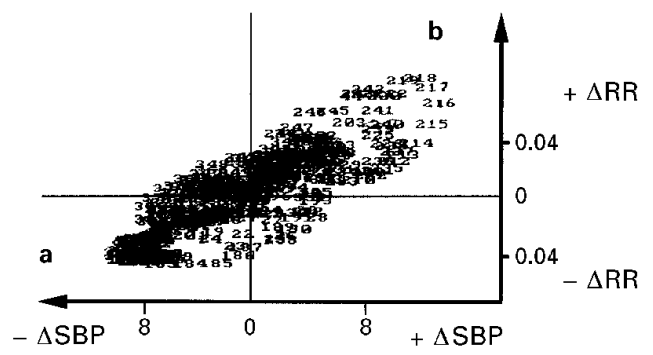

C
(1)

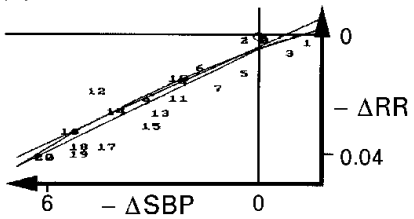

$\mathrm{Sp}-\mathrm{BRS}=6.41$

$$
(\mathrm{r}=0.89)
$$

(2)

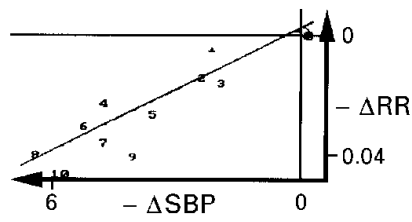

$\mathrm{Sp}-\mathrm{BRS}=6.83$

$(r=0.89)$
Figure 1 Example of recording and baroreflex sensitivity calculation in a preterm neonate. (A) Illustration of the $S p-S B P(\mathrm{~mm} \mathrm{Hg}$ ) and heart rate (beats/min) simultaneously recorded in one of the 14 preterm neonates studied. Two periods of activation of the baroreflex are delimited by the vertical dashed lines. (B) When calculated for the whole 4 minute period (more than 300 points), the slope of the regression line indicating the $S p-B R S$ was $4.50 \mathrm{~ms} / \mathrm{mm} \mathrm{Hg}(r=0.85)$. (C) During the period (1), Sp-BRS was 6.41 $\mathrm{ms} / \mathrm{mm} \mathrm{Hg}(r=0.89)$ and during the period (2) $S p-B R S$ was $6.83 \mathrm{~ms} / \mathrm{mm} \mathrm{Hg}(r=0.89)$. babies, whose gestational ages were, respectively, 28 and 30 weeks, both had frequent episodes of severe bradycardia. Treatment with prantal significantly decreased their baroreflex sensitivity values, from $11.61(1.90) \mathrm{ms} / \mathrm{mm}$ $\mathrm{Hg}$ to $3.92(1.30) \mathrm{ms} / \mathrm{mm} \mathrm{Hg}$ in the first one, and from 16.79 (1.67) $\mathrm{ms} / \mathrm{mm} \mathrm{Hg}$ to 1.58 $(0.16) \mathrm{ms} / \mathrm{mm} \mathrm{Hg}$ in the other.

\section{Discussion}

In adults several non-perturbational methods have already been described to estimate Sp-BRS - in humans ${ }^{4}$ as well as in animals. ${ }^{78}$ These studies showed that pharmacological baroreflex sensitivity is strongly positively correlated with $\mathrm{Sp}$-BRS, although Sp-BRS operates only in a small linear portion of the sigmoidal curve estimated from pharmacological estimation of baroreflex. ${ }^{8}$ Classic pharmacological methods of assessment of baroreflex sensitivity are limited by their perturbational character and by theoretical considerations, such as modifications of barosensitive areas induced by drugs. ${ }^{3}$ The Sp-BRS method is based on the assumption that spontaneous fluctuations in systolic blood pressure also stimulate baroreceptors, inducing fluctuations in RR intervals by baroreflex mechanisms.

As stated previously, very little is known about the baroreflex sensitivity in human neonates, and no "normal" values are available for comparison. Although it was not possible in this study to compare Sp-BRS with the sigmoidal curves obtained with the drug induced method to validate the spontaneous method, there are several arguments that make our results consistent. First, the values of baroreflex sensitivity were not significantly different when

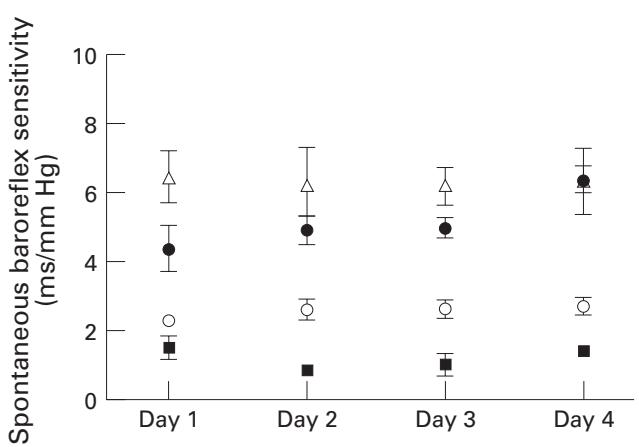

Figure 2 Individual reproducibility of $S p-B R S$ over time (four consecutive days) in four neonates. The evolution in the first neoante is represented by squares, the second by open circles, the third by closed circles, and the fourth by open triangles. 
calculated from the sequences of bradycardia/ hypertension $(+\Delta \mathrm{RR} /+\Delta \mathrm{SBP})$ or tachycardia/ hypotension $(-\Delta R R /-\Delta S B P)$, both in the preterm and in the term neonates (table 2). Secondly, the individual reproducibility of $\mathrm{Sp}$-BRS values obtained in four neonates over four consecutive days (fig 2) also corroborate our findings. The small average range of Sp-BRS within each neonate indicated day-today variability. We also observed similar $\mathrm{Sp}$-BRS values in three pairs of twins, suggesting that the maturation of their autonomic nervous system was similar. Finally, the high $\mathrm{Sp}$-BRS values fell after treatment with prantal, an anticholinergic quaternary ammonium methylsulfate, in two babies.

We did not correlate the information on Sp-BRS with the analysis of heart rate variability. Although both techniques measure parasympathetic activity, heart rate variability and baroreflex sensitivity are not redundant. ${ }^{8}$ A study by Barron and Lesh suggested that the two methods measure different aspects of parasympathetic activity. ${ }^{9}$ Indeed, heart rate variability primarily reflects tonic vagal activity, whereas baroreflex sensitivity measures predominantly reflex vagal activity. ${ }^{10}$

The Sp-BRS method has numerous advantages. First, it does not require the use of drugs or neck chamber apparatus. Second, it measures baroreflex sensitivity in the normal physiological range over a period of time rather than during brief and extreme perturbations induced by pharmacological methods. Third, the $\mathrm{Sp}$-BRS method is non-invasive when used with non-invasive continuous systolic blood pressure monitoring such as Finapres. Thus it can be used frequently for serial measurements - for example, to study the ontogeny of baroreflex sensitivity in neonates.

This study has two principal limitations for the assessment of Sp-BRS in neonates. The first is the limited range of fluctuation in systolic blood pressure and heart rate in resting conditions in some of the neonates. This is why Sp-BRS could only be measured in 14 out of 18 preterm neonates and in five out of seven term neonates. The second is a methodological limitation: to measure baroreflex sensitivity, we selected short periods with important spontaneous variations in systolic blood pressure, inducing $R R$ variations consistent with a baroreflex mechanism. By selecting periods with high baroreflex activity, we may have introduced a bias and, consequently, may have overestimated baroreflex sensitivity. A more objective method to evaluate $\mathrm{Sp}$-BRS has been described recently by Cerutti et $a .^{8}{ }^{8}$ This method relies on the determination of the statistical dependence of pairs of mean arterial pressure (MAP) and heart rate values. Only the pairs shown to be related to the baroreflex activity $(+\Delta \mathrm{RR} /+\Delta \mathrm{MAP} ;-\Delta \mathrm{RR} /-\Delta \mathrm{MAP})$ are taken into account for calculation of baroreflex sensitivity. Baroreflex sensitivity is then evaluted using the slope of the regression line between these selected MAP and heart rate values, with each pair (MAP, heart rate) being weighed according to its degree of dependence and its frequency of observation.
There is extensive published data which suggest that disorders of the autonomic nervous system, including cardiorespiratory function, may be involved in sudden infant death syndrome (SIDS) and apparent lifethreatening events. The maturation of the control of the cardiovascular system by the autonomic nervous system continues after birth. The degree of maturation may have an influence on the susceptibility to malignant arrhythmias during the first months of life. Therefore, a more comprehensive knowledge of the time course of postnatal autonomic nervous system maturation may be relevant to understanding the mechanisms involved in SIDS.

Until now, investigation of the autonomic nervous system has been confined to oculocardiac reflex and heart rate responses. Parasympathetic function was tested by means of the heart rate beat-to-beat variability. Baroreflex sensitivity is an alternative and quantitative approach to study autonomic nervous system activity. The ontogeny of baroreflex sensitivity might be helpful to the study of autonomic nervous system maturation. The non-invasive method that we describe here might help to resolve the discrepancies between previous results on autonomic nervous system maturation.

Indeed, all the previous studies on the ontogeny of baroreflex sensitivity, performed in animal models, have yielded conflicting results. Arterial baroreflex responses have been demonstrated during fetal and postnatal life. However, the evolution of baroreflex sensitivity with maturation is still debated. Several authors have described a reduced heart rate response to alterations in systolic blood presure in neonates compared with adult animals, suggesting that baroreflex sensitivity increases postnatally with maturation. ${ }^{11-13}$ However, others have found that baroreflex sensitivity is greater in the fetus than in the neonate and that it decreases with maturation. ${ }^{1-16}$ One study showed that baroreflex sensitivity is lower at birth than in adult life. ${ }^{17}$

From our study, it seems that Sp-BRS is reduced in preterm compared with term neonates-respectively, 4.07 (2.19) $\mathrm{ms} / \mathrm{mm} \mathrm{Hg}$ and 10.23 (3.07) $\mathrm{ms} / \mathrm{mm} \mathrm{Hg}$, suggesting an increase in baroreflex sensitivity with maturation. This difference indicates a vagal tone that is stronger in term neonates than in preterm neonates, probably because the efferent control system is underdeveloped in preterms. One possibility is that vagal innervation may not exert its full influence on the sinus node in preterm neonates.

In conclusion, this study shows that it is possible to assess non-invasively cardiac baroreflex sensitivity from spontaneous systolic blood pressure and RR variations in neonates. It may prove a useful method to describe the developmental course of baroreflex sensitivity in children, from preterm, term neonates to older infants, and to compare it with the vagal tone measured by spectral analysis of sinusal RR interval. Furthermore, Sp-BRS may be a useful 
tool to assess autonomic nervous system maturity in infants at risk of SIDS.

This study was supported by a grant 95/6-H (Projet Hospitalier de Recherche clinique 1995) from the Ministère de la Santé, Paris, France.

1 Vanoli E, Adamson Ph B. Baroreflex sensitivity: Methods, mechanisms, and prognostic value. Pacing Clin Electrophys 1994;17:434-45.

2 Smyth HS, Sleight P, Pickering GW. Reflex regulation of arterial pressure during sleep in man: a quantitative method for assessing baroreflex sensitivity. Circ Res 1969 24:109-2

3 Peveler RC, Bergel DH, Robinson JL, Sleight P. The effects of phenylephrine upon arterial pressure, carotid sinus radius and baroreflex sensitivity in the conscious greyhound. Clin Sci Lond 1983;64:455-61.

4 Parlow J, Viale JP, Annat G, Hughson R, Quintin L. Spontaneous cardiac baroreflex in humans. Comparison with drug-induced responses. Hypertension 1995;25:1058-68.

5 Parati G, Omboni S, Frattola A, Di Rienzo M, Zanchetti A, Mancia G. Dynamic evaluation of the baroreflex in ambulant subjects. In : di Rienzo M, et al, eds. Blood pressure and heart rate variability. IOS Press, 1992: 123-37.

6 Droun E, Gournay V, Calamel J, Mouzard A, Rozé JC. Instantaneous non-invasive measurement of blood presInstantaneous non-invasive measurement of b
sure in the newborn. Pediatr Res 1996;39:206A.

7 Frankel RA, Mettting PJ, Britton SL. Evaluation of spontaneous baroreflex sensitivity in conscious dogs. $\mathcal{F}$ Physiol

8 Cerutti C, Ducher M, Lantelme P, Gustin MP, Paultre C. Assessment of spontaneous baroreflex sensitivity in rats a new method using the concept of statistical dependence. Am F Physiol 1995;268:R382-R8.

9 Barron H, Lesh M. Autonomic nervous system and sudden cardiac death. I Am Coll Cardiol 1996;27:1053-60.

10 Schwartz PJ, La Rovere MT, Vanoli E. Autonomic nervous system and sudden cardiac death: experimental basis and clinical observations for post-myocardial infarction risk clinical observations for post-myocardial infarction
stratification. Circulation 1992;85(Supp1 I): I77.

11 Gootman PM.Developmental aspects of reflex control of the circulation. In: Zucker IH, Gilmore JP, eds. Reflex control of the circulation. Boca Raton, FL: CRC Press, 1991: 965-1027.

12 Ismay MJA, Lumbers R, Stevens AD. The action of angiotensin II on the baroreflex response of the conscious ewe and the conscious fetus. F Physiol Lond 1979;288: 467-79.

13 Shinebourne EA, Vapaavouri EK, Williams MA, Heyman MA, Rudolph AM. Development of baroreflex activity in unanesthetized fetal and neonatal lambs. Circ Res 1972; unanesthetized

14 Maloney JE, Cannata JP, Dowling MH, Else W, Ritchie B. Baroreflex activity in conscious fetal and newborn lambs. Biol Neonate 1977;31:340-50.

15 Palmisano BW, Cliffrod PS, Coon RL, Seagard JL, Hoffmann RG, Kampine JP.Development of baroreflex control of heart rate in swine. Pediatr Res 1989;27:148-52.

16 Segar JL, Hajduczok G, Smith BA, Merrill DC, Robillard $\mathrm{JE}$. Ontogeny of baroreflex control of renal sympathetic nerve activity and heart rate. Am f Physiol 1992;263: H1819-H26.

17 Young M. Responses of the systemic circulation of the newborn infant. Br Med Bull 1966;22:70-2. 